\title{
Long-Term Safety and Efficacy of Recombinant Coagulation Factor IX Albumin Fusion Protein (rIX-FP) in Previously Treated Pediatric Patients with Hemophilia B: Results from a Phase 3b Extension Study
} \author{
Wilfried Seifert $^{7}$ Elena Santagostino ${ }^{8}$ \\ ${ }^{1}$ The Israeli National Hemophilia Center, Sheba Medical Center, Tel \\ Hashomer, Sackler Medical School, Tel Aviv University, Israel \\ 2 APHM, Pediatric Hematology Oncology Department, Children Hospital La \\ Timone \& Aix Marseille University, INSERM, INRA, C2VN, Marseille, France \\ ${ }^{3}$ Department of Pediatrics, Medical University of Vienna, Vienna, Austria \\ ${ }^{4}$ CRC Coagulation Research Centre GmbH, Duisburg, Germany \\ ${ }^{5}$ Centre de Traitment des Hémophiles, Hôpital Bicetre, Paris, France \\ ${ }^{6}$ CSL Behring, King of Prussia, United States \\ ${ }^{7}$ CSL Behring, Marburg, Germany \\ 8 Fondazione IRCCS Ca' Granda, Ospedale Maggiore Policlinico, Angelo \\ Bianchi Bonomi Hemophilia and Thrombosis Center, Milan, Italy
}

Gili Kenet ${ }^{1}$ Hervé Chambost ${ }^{2}$ Christoph Male ${ }^{3}$ Susan Halimeh ${ }^{4}$ Thierry Lambert ${ }^{5}$ Yanyan Li ${ }^{6}$

\begin{abstract}
Address for correspondence Elena Santagostino, MD, PhD, Fondazione IRCCS Ca' Granda, Ospedale Maggiore Policlinico, Angelo Bianchi Bonomi Hemophilia and Thrombosis Center, Via Pace 9, 20122 Milano, Italy (e-mail: e_santagostino@hotmail.com).
\end{abstract}

Thromb Haemost 2020;120:599-606.

\begin{abstract}
Keywords

- rIX-FP

- factor IX

- hemophilia B

- prophylaxis

- recombinant fusion protein
\end{abstract}

Introduction A phase $3 \mathrm{~b}$ extension study evaluated the long-term safety and efficacy of a recombinant fusion protein-linking coagulation factor IX (FIX) with albumin (rIX-FP) for the routine prophylaxis and on-demand treatment of bleeding in pediatric hemophilia $B$ patients. Methods Previously treated patients aged $<12$ years with moderate to severe hemophilia B enrolled in a 3-year extension study following a phase 3 pivotal study in which they received weekly rIX-FP prophylaxis. In the extension study, they could maintain or extend their prophylaxis interval to every 10 or 14 days if they were well controlled on the 7-day regimen.

Results Compared with their initial regimen, by the end of the study, dosing intervals were the same, extended, and shortened in 16,4 , and 4 patients, respectively. Very low annualized spontaneous bleeding rates (AsBRs) were observed; median AsBR was 0.0 for the 7- and 10-day regimens, and 1.1 for the 14-day regimen. The 7- and 14-day regimens were comparable in preventing spontaneous bleeds; mean (95\% confidence interval) difference in AsBR of -1.2 (-2.6 to 0.3) bleeding episodes/year/subject. Overall, $96 \%$ of bleeding episodes were successfully treated with one or two injections of rIX-FP. Patients on a 14-day regimen maintained a mean steady-state trough FIX level of $>7.2 \mathrm{IU} / \mathrm{dL}$. No patient developed an inhibitor.

Conclusion This extension study demonstrated the long-term safety and efficacy of weekly rIX-FP in pediatric patients. Additionally, it showed that adequate bleed protection can be achieved with 10- or 14-day rIX-FP regimens in selected pediatric patients while maintaining safety. received

September 10, 2019

accepted

January 15, 2020
DOI https://doi.org/

10.1055/s-0040-1705116.

ISSN $0340-6245$. (c) 2020 Georg Thieme Verlag KG Stuttgart · New York
License terms

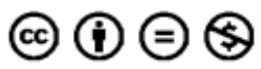




\section{Introduction}

Hemophilia B is an X-linked recessive genetic disorder caused by a functional deficiency of coagulation factor IX (FIX). ${ }^{1}$ It is the second most common type of hemophilia with an incidence of approximately 1 in 30,000 people. ${ }^{2}$ An absence or dysfunction of FIX is associated with spontaneous bleeding; particularly in patients with a moderate-to-severe disease phenotype ( $<5 \mathrm{IU} / \mathrm{dL}$ FIX activity). Spontaneous or traumatic bleeding into the muscles, soft tissue, or joints may result in crippling arthropathy, disability, and reduced quality of life. ${ }^{1}$ Reduction of bleeds and preservation of normal musculoskeletal function is the primary goal of routine prophylaxis therapy. ${ }^{3}$

Prophylaxis with FIX replacement therapy is recommended in all children with severe hemophilia. ${ }^{1,4}$ The short half-life of FIX (18-24 hours) means frequent infusions with standard half-life FIX concentrates are required to maintain target trough levels. ${ }^{1}$ There is limited evidence for a target trough level in hemophilia B, but a study in hemophilia A suggested that maintaining trough levels above $15 \%$ could eradicate joint bleeding. ${ }^{5}$ A study in hemophilia B patients has shown that increased time spent below a $1 \%$ FIX activity level is associated with an increase in total bleeding events. ${ }^{6}$

The availability of extended half-life (EHL) FIX products may decrease treatment burden by extending dosing intervals while maintaining higher trough levels than standard FIX products. $^{7}$ Pediatric patients have additional considerations when choosing a treatment regimen, compared with adult patients, such as higher FIX clearance, higher activity levels, a higher number of traumatic bleeds, and difficulty with venous access. ${ }^{8}$ Young patients also require assistance with infusions and so extended dosing intervals and reduced infusion frequency may decrease both patient and caregiver burden.

A recombinant fusion protein genetically linking human coagulation FIX with human albumin, rIX-FP, (IDELVION, CSL Behring) has been developed. ${ }^{9}$ Recombinant coagulation factor IX albumin fusion protein (rIX-FP) has previously shown an improved pharmacokinetic profile, with an approximately fivefold longer half-life compared with standard rFIX products. ${ }^{10}$ The efficacy and safety of rIX-FP has also been demonstrated with weekly prophylaxis in previously treated pediatric patients treated with rIX-FP for a mean exposure time of over 12 months. ${ }^{10}$

As part of the PROLONG-9FP clinical trial program, an extension study was undertaken to evaluate the long-term safety and efficacy of rIX-FP in routine prophylaxis. Here, we report the findings of this phase $3 \mathrm{~b}$ extension study in previously treated patients $<12$ years of age with prophylaxis dosing intervals of up to 14 days. Findings in adult and adolescent patients $\geq 12$ years of age will be reported separately. ${ }^{11}$

\section{Methods}

\section{Study Conduct}

The study was approved by the institutional review board/ethics committee at each participating center, registered at www.clinicaltrials.gov (NCT02053792), and performed in accordance with good clinical practice, the
Declaration of Helsinki, ${ }^{12}$ and local regulatory requirements. Written informed consent was obtained from the parent/guardian of the patient and informed assent from the patient. Consent could be withdrawn at any time.

\section{Study Participants}

Male patients aged $<12$ years with moderate-to-severe hemophilia B (FIX activity $\leq 2 \%$ ), previously treated with FIX products, and with no personal or family history of FIX inhibitor formation or detectable inhibitors at screening were eligible for enrollment. Eligible patients had completed a phase 3 pediatric study (NCT01662531) ${ }^{10}$ or could enter the study after undergoing nonemergency surgery with rIX-FP.

\section{Study Design}

In this phase $3 \mathrm{~b}$, prospective, multicenter extension study, enrolled subjects were assigned to a 7-day (25-50 IU/kg), 10day, or 14-day $(50-75 \mathrm{IU} / \mathrm{kg}$ ) regimen; this was based on treatment success during the phase 3 lead-in study (35-75 IU/kg rIX-FP weekly prophylaxis) (NCT01662531) and/or investigator and subject preference for the first 6 months of the study. At each 6-month interval, the investigator could change the regimen based on an assessment of efficacy in the patient, safety, treatment compliance, and/or preference. Treatment with rIX-FP continued for approximately 30 months to achieve a minimum of 100 exposure days (EDs). On-demand treatment with 35 to $75 \mathrm{IU} / \mathrm{kg}$ rIX-FP was administered for bleeding episodes.

\section{Study Objectives}

The primary endpoint of this study was the total number of subjects who developed inhibitors against FIX. Secondary endpoints were to evaluate the efficacy of rIX-FP with various regimens by assessing annualized bleeding rate (ABR), spontaneous ABR (AsBR), and joint ABR (AjBR) for treated bleeds. Bleeding episodes and the number of infusions required for hemostasis were recorded and analyzed. Monthly consumption of rIX-FP during routine prophylaxis, the incidence of rIX-FP related and nonrelated adverse events (AEs), and steady-state trough FIX activity levels were also evaluated.

\section{Safety Assessments}

Patients who received at least one dose of rIX-FP were assessed for inhibitor incidence. Inhibitors were titrated by the Bethesda method according to the Nijmegen modification $^{13}$ and only patients who had two consecutive positive tests $(\geq 0.6 \mathrm{BU} / \mathrm{mL}$ ) were classified as having an inhibitor.

The incidence of antibodies against rIX-FP and Chinese hamster ovary $(\mathrm{CHO})$ cell-derived proteins were determined using direct binding enzyme-linked immunoabsorbent assays, as previously described. ${ }^{14}$ The frequency, type, and severity of AEs, as well as their relationship to rIX-FP; local tolerability at the injection site; laboratory safety parameters; and vital signs were also assessed.

\section{Steady-State Trough FIX Activity}

FIX activity was measured at major bleeding events and scheduled assessments only at a central laboratory using a 
validated one-stage clotting method with Pathromtin SL (Siemens Healthcare Diagnostics, Marburg, Germany) as an activator agent. Steady-state trough FIX activity was assessed at months $6,12,18,24,30,36$, and 42 . Steady-state trough FIX activity included measurements before a third consecutive dose on the 10- or 14-day regimens or before a fourth consecutive dose on the 7-day regimen. If there were any unscheduled rIX-FP doses administered (e.g., to treat a bleed), the FIX activity measurements within 28 days of the unscheduled dose were excluded because the washout period for rIX-FP is approximately 5 half-lives. FIX activity was measured.

\section{Efficacy Assessments}

$A B R, A s B R$, and $A j B R$ were calculated for each regimen. The differences in mean $A B R$ and AsBR between the 14-day regimen and the 7-day regimen (combined from the leadin and extension study) were calculated. Bleeding episodes were summarized by severity, type, site, and location. Treatment of mild and moderate bleeding episodes with rIX-FP was considered successful if hemostasis was achieved with one or two injections.

Target joints were defined as joints where three or more treated spontaneous bleeds occurred within a 6-month period and were considered resolved if there were $\leq 2$ spontaneous bleeds into the same joint within the subsequent 12-month period.

Consumption, by treatment modality, was reported as number of injections per subject, IU/kg per subject and IU $/ \mathrm{kg}$ per event.

\section{Statistical Analysis}

Subjects receiving at least one dose of rIX-FP were included in the safety analysis. Data were included for patients during the prophylaxis treatment period; safety data recorded during the surgical substudy were not included. Data from the surgical substudy will be described separately. ABR, AsBR, and AjBR were calculated for each regimen for all patients who had been on the regimen for at least 12 weeks; the same patient may contribute data to more than one regimen based on the duration of treatment received. A crude rate was calculated per regimen with an exact two-sided 95\% confidence interval (CI) assuming a Poisson-distributed event rate. ABRs and AsBRs were compared for subjects who received both the 7and 14-day prophylaxis regimen.

Data were summarized using descriptive statistics for continuous data, or frequencies and percentages for categorical data.

\section{Results}

\section{Study Population}

Twenty-four males previously treated patients (FIX activity $\leq 2 \%$ ) were enrolled in the extension study from the pivotal phase 3 study, in which they had received 7-day prophylaxis with rIX-FP; no patients were enrolled subsequent to surgery. In the extension study, seven patients were in the younger age group ( $<6$ years), and 17 patients in the older
Table 1 Patient characteristics

\begin{tabular}{|l|l|}
\hline & $n=24$ \\
\hline Age $(\mathrm{y})$, median (range) & $7.0(2-11)$ \\
\hline$<6(n=7)$ & $4.0(2-5)$ \\
\hline$\geq 6$ to $<12(n=17)$ & $9.0(6-11)$ \\
\hline Height, cm & $124.9(19.3)$ \\
\hline Weight, kg & $28.6(14.6)$ \\
\hline Race, $n(\%)$ & \\
\hline White & $23(95.2)$ \\
\hline Black & $1(4.8)$ \\
\hline Asian & 0 \\
\hline Geographic region & \\
\hline North America & 1 \\
\hline Europe & 17 \\
\hline Middle East & 4 \\
\hline Asia & 0 \\
\hline Africa & 0 \\
\hline Oceania & 2 \\
\hline
\end{tabular}

age group ( $\geq 6-<12$ years). In the phase 3 study, 11 of these patients were in the younger age group, and 13 in the older age group. Participants were aged between 2 and 11 years of age with a median (range) of $4.0(2-5)$ years in the younger age group and 9.0 (6-11) years in the older age group; patient demographics are provided in - Table 1 . This extension study was conducted between February 2014 and October 2018 at 14 sites in nine countries; patients were treated with rIX-FP for a mean (standard deviation [SD]) 37.1 (9.8) months.

In the extension study, based on the investigator's decision, patients were assigned to 7 - $(n=18), 10$ - $(n=2)$, or 14 -day $(n=4)$ prophylaxis. - Fig. 1 summarizes patient disposition and study flow. No patients had target joints at study entry. In total, 23 patients completed the study and one withdrew; the patient suffered from two traumatic bleeds while on a 14-day regimen $(75 \mathrm{IU} / \mathrm{kg})$ and a further two traumatic bleeds when his prophylaxis interval was reduced to 7 days ( $50 \mathrm{IU} / \mathrm{kg}$ ). None of these four traumatic bleeding episodes occurred in joints. The patient withdrew after approximately 2.5 months on the 7-day dosing interval. No trough FIX activity levels relative to these bleeding episodes were available for this patient. Across both the pivotal and extension studies, all subjects (100\%) achieved $\geq 50$ EDs and 23 (95.8\%) achieved $\geq 100$ EDs.

\section{Safety}

During the extension study, no patient developed inhibitors to FIX or antibodies to rIX-FP or CHO cell proteins. A total of 215 treatment-emergent AEs (TEAEs) were reported in 23 (95.8\%) patients during the study; the majority (95.8\%) was mild or moderate in severity and no TEAEs were considered related to study drug. The most commonly reported TEAEs were pyrexia (15 events in 10 patients), nasopharyngitis (15 events in 8 patients), arthralgia (10 events in 6 patients), and headache ( 9 events in 5 patients). 




Fig. 1 Patient enrollment and study flow diagram. Figure demonstrates only initial and final treatment regimens; patients may have switched regimens more than once during the study period. *Includes one patient with an initial 7-day dosing regimen, who switched to 10-, 14-, and 10day dosing regimens, before switching back to a 7-day regimen; includes another patient with an initial 7-day dosing regimen, who switched to a 10-day dosing regimen, before switching back to a 7-day regimen. ${ }^{\dagger}$ Includes one patient with an initial 7-day dosing regimen, who switched to 14 day, 10-day, and 14-day regimens, before switching back to a 10-day regimen. ${ }^{\ddagger}$ Includes a patient with an initial 7-day dosing regimen, who switched to a 10-day regimen, before finishing the study on a 14-day regimen.

Fourteen treatment-emergent serious AEs (SAEs) were observed in seven (29.1\%) patients including four mild, three moderate, and seven severe events. All SAEs required hospitalization and included head trauma (four events), anemia (two events) and one incident of acute appendicitis; moderate hemarthrosis 1day post right knee arthrocentesis (the associated major traumatic bleeding episode was treated with four doses of rIX-FP, with hemostatic efficacy assessed as excellent; this bleeding episode has been considered as part of the surgical substudy), muscle injury, hematuria, arthralgia, life-threatening extradural hematoma due to a fall, jaw abscess, and epistaxis. No SAEs were considered related to study drug. No anaphylactic reactions or thromboembolic events were reported.

\section{Prophylaxis Dosing Intervals}

The majority of patients were able to maintain their prophylactic dosing interval during the study. Compared with their initial regimen, by end of study, 4 (16.7\%) patients had extended their dosing interval, 16 (66.6\%) had not changed, and $4(16.7 \%)$ had shortened their dosing interval. During the course of the study, a total of eight patients were prescribed a prophylaxis regimen of every 14 days including two patients who switched from a 7-day regimen to a 14-day regimen and two patients that maintained a 14-day regimen throughout the study. This also includes two patients who switched from their initial 14-day regimen back to a $10-(n=1)$ or 7-day $(n=1)$ regimen. Additionally, two patients with initial 7-day regimens switched to a 14 -day regimen (via a 10-day regimen $[n=1])$ and eventually reduced their dosing frequency to a 10- $(n=1)$ or 7-day $(n=1)$ regimen. At the end of the study, 17, 3 and 4 patients were on a 7-, 10-, and 14-day regimen, respectively ( - Fig. $\mathbf{1}$ ). In the younger age group, six of seven patients were on 7-day prophylaxis at the end of the study and one patient remained on 14-day prophylaxis for the duration of the study.

\section{Consumption}

Mean (SD) monthly prophylactic consumption was 212.4 (34.4), 198.6 (44.8), and 154.5 (11.3) IU/kg for the 7-, 10-, and 14-day prophylaxis regimens, respectively ( - Table 2). Mean (SD) overall monthly consumption, including the treatment of bleeding events, was 231.2 (42.0), 224.2 (58.4), and 185.4 (24.0) IU/kg for the 7-, 10-, and 14-day regimens, respectively. A total of 3,269 prophylactic infusions of rIX-FP were administered intravenously during the extension study. The mean (SD) number of infusions per patient per year were

Table 2 Summary of monthly consumption across recombinant coagulation factor IX albumin fusion protein (rIX-FP) dosing regimens

\begin{tabular}{|l|l|l|l|}
\hline & $\begin{array}{l}\text { 7 day } \\
(\boldsymbol{n}=\mathbf{2 1})\end{array}$ & $\begin{array}{l}\text { 10 day } \\
(\boldsymbol{n}=\mathbf{8})\end{array}$ & $\begin{array}{l}\mathbf{1 4} \text { day } \\
(\boldsymbol{n}=\mathbf{8})\end{array}$ \\
\hline Prophylaxis & & & \\
\hline $\begin{array}{l}\text { Monthly consumption, } \\
\text { IU/kg }\end{array}$ & $\begin{array}{l}212.4 \\
(34.4)\end{array}$ & $\begin{array}{l}198.6 \\
(44.8)\end{array}$ & $\begin{array}{l}154.5 \\
(11.3)\end{array}$ \\
\hline $\begin{array}{l}\text { Injections per patient } \\
\text { per month }\end{array}$ & $4.3(0.1)$ & $3.0(0.1)$ & $2.1(0.1)$ \\
\hline Overall & & & \\
\hline $\begin{array}{l}\text { Monthly consumption, } \\
\text { IU/kg }\end{array}$ & 231.2 & 224.2 & $\begin{array}{l}185.4 \\
(24.0)\end{array}$ \\
\hline $\begin{array}{l}\text { Injections per patient } \\
\text { per month }\end{array}$ & $4.6(0.4)$ & $3.4(0.3)$ & $2.6(0.3)$ \\
\hline
\end{tabular}

Note: Data given as mean (standard deviation).

${ }^{a}$ Overall data include dosing for prophylaxis and additional infusions for bleeding events. 
43.7 (9.6) and there were 155.0 (55.2) EDs per patient overall. As expected, the 14-day prophylaxis regimen also had the lowest average monthly number of prophylactic infusions, 2.1 (0.1), compared with the 7- and 10-day regimens, 4.3 (0.1) and 3.0 (0.1), respectively. Including additional infusions for bleeding events, patients on the 14-day regimen still had the lowest average monthly number of infusions, 2.6 (0.3), compared with the 7- and 10-day regimens, $4.6(0.4)$ and $3.4(0.3)$, respectively. Median (range) total dose per infusion during prophylaxis was $49.0(22-86) \mathrm{IU} / \mathrm{kg}$ for the 7day regimen, 74.0 (40-82) $\mathrm{IU} / \mathrm{kg}$ for the 10-day regimen, and 73.7 (56-82) IU/kg for the 14-day regimen.

\section{Steady-State Trough FIX Activity}

Mean (SD) steady-state trough FIX activity levels (\%) were 15.1 (4.1), 8.8 (5.7), and 7.2 (2.9) for 7-, 10-, and 14-day regimens, respectively. Mean (SD) steady-state trough FIX activity levels (\%) were 15.3 (3.8), 8.0 (2.5) in the younger group, and 15.0 (4.5) and 6.9 (3.0) in the older group for 7and 14-day regimens, respectively.

\section{Efficacy}

The efficacy of prophylactic rIX-FP treatment was demonstrated with low AsBRs achieved across all regimens (-Table $\mathbf{3}$ ). Median AsBR was 0.0 for the 7- and 10-day regimens, and 1.1 for the 14-day regimen. Mean (SD) AsBR was 0.6(1.3), 1.6 (2.5), and 1.7 (2.0) for the 7-, 10-, and 14-day regimens, respectively. The mean $(95 \% \mathrm{CI})$ difference (7-day minus 14-day) in AsBR was -1.2 ( -2.6 to 0.3 ) bleeding episodes per year per subject and $-2.9(-5.3$ to -0.5$)$ in total ABR. Fourteen (66.7\%), five $(62.5 \%)$, and three $(37.5 \%)$ patients on 7 -, 10 - or 14 -day regimens experienced zero spontaneous bleeding episodes, respectively. Additionally, nine (42.9\%), three (37.5\%), and three $(37.5 \%)$ patients on $7-, 10-$, or 14 -day regimens experienced zero joint bleeding episodes, respectively.

Two patients, aged 5 and 7 years, developed a total of three target joints during the extension study. Both patients were previously on prophylaxis with standard half-life FIX prior to entering the clinical study program. One patient developed a target joint in the elbow while on a 10-day regimen and experienced 10 bleeds in this joint over a 6-month period; he had previously experienced a traumatic bleed in this joint during the phase 3 lead-in study. Following this bleed, his dose was adjusted from 55 to $76 \mathrm{IU} / \mathrm{kg}$ every 10 days; he was then switched to $70 \mathrm{IU} / \mathrm{kg}$ every 7 days due to further bleeding events. He later developed a target joint in the ankle after a spontaneous bleeding episode while on a 7-day regimen; he experienced a total of three bleeds in this joint over a 4.5-month period. After resolution of this target joint, his dose was reduced to $45 \mathrm{IU} / \mathrm{kg}$ every 7 days until the end of the study. The second patient developed a target joint in the knee following a traumatic bleed while on a 7-day regimen. He had previously experienced no bleeds in this joint during the phase 3 lead-in study, and reported three bleeds in this joint over a 4-month period in the extension study. Following the initial bleeding event, his dose was adjusted from 41 to $48 \mathrm{IU} / \mathrm{kg}$ every 7 days, and he remained on this higher dose until the end of the study. Over the course of the study, while on 7- or 10-day prophylaxis with rIX-FP, all target joints resolved in both patients.

During the extension study, a total of 301 bleeding episodes were reported that required treatment, including 78 unclassified events. Of 71 spontaneous bleeds requiring treatment, there were 35,13 , and 23 bleeds reported in patients receiving a 7- $(n=21), 10$ - $(n=8)$, or 14-day $(n=8)$ regimen. Of 152 traumatic bleeding episodes requiring treatment, 102,16 , and 34 of these were reported in patients receiving a 7- $(n=21)$, 10 - $(n=8)$, or 14-day $(n=8)$ prophylaxis regimen. Successful treatment with one or two injections of rIX-FP was reported in $96 \%$ of bleeds requiring treatment.

\section{Discussion}

Data from this extension study are consistent with data previously reported in a phase 3 trial of rIX-FP in pediatric patients. ${ }^{10}$ The phase 3 trial demonstrated the safety and efficacy of 7-day rIX-FP prophylaxis in pediatric patients. This extension study has shown that rIX-FP is well tolerated and efficacious with an extended prophylactic dosing interval of up to 14 days in selected pediatric patients. Providing further validation for rIX-FP's favorable safety profile, no

Table 3 Efficacy of recombinant coagulation factor IX albumin fusion protein (rIX-FP) in prophylaxis regimens

\begin{tabular}{|c|c|c|c|c|}
\hline \multicolumn{2}{|c|}{ Regimen } & \multirow{2}{*}{$\begin{array}{l}7 \text { day }(n=21)^{a} \\
0.0(0.0-0.5)\end{array}$} & \multirow{2}{*}{$\begin{array}{l}10 \text { day }(n=8) \\
0.0(0.0-2.8)\end{array}$} & \multirow{2}{*}{$\begin{array}{l}14 \text { day }(n=8) \\
1.1(0.0-3.4)\end{array}$} \\
\hline AsBR & Median (Q1-Q3) & & & \\
\hline & Mean (SD) & $0.6(1.3)$ & $1.6(2.5)$ & $1.7(2.0)$ \\
\hline & $n$ (\%) with zero bleeds & $14(66.7)$ & $5(62.5)$ & $3(37.5)$ \\
\hline \multirow[t]{3}{*}{$A B R$} & Median (Q1-Q3) & $2.0(0.7-4.7)$ & $3.5(0.8-6.7)$ & $5.6(2.0-6.9)$ \\
\hline & Mean (SD) & $3.3(3.6)$ & $4.0(3.6)$ & $4.7(3.1)$ \\
\hline & $n$ (\%) with zero bleeds & $3(14.3)$ & $2(25.0)$ & $1(12.5)$ \\
\hline \multirow[t]{3}{*}{ AjBR } & Median (Q1-Q3) & $0.6(0.0-2.6)$ & $2.0(0.0-3.8)$ & $2.6(0.0-3.3)$ \\
\hline & Mean (SD) & $1.8(2.9)$ & $2.5(2.8)$ & $2.0(1.7)$ \\
\hline & $n(\%)$ with zero bleeds & $9(42.9)$ & $3(37.5)$ & $3(37.5)$ \\
\hline
\end{tabular}

Abbreviations: ABR, annualized bleeding rate; AjBR, annualized joint bleeding rate; AsBR, annualized spontaneous bleeding rate; Q1, 1st quartile; Q3, 3rd quartile; SD, standard deviation.

${ }^{a} A B R s$ include only subjects who have been on each regimen for at least 12 weeks $(n=20)$. 
inhibitors were reported and no adverse events were deemed related to treatment. Extended dosing intervals are of significant clinical interest, particularly in young children with challenging venous access.

During rIX-FP prophylaxis, FIX trough levels were maintained well above the recommended minimum of $1 \%$ across all dosing regimens. ${ }^{1}$ Although this is a commonly accepted goal, recent data have suggested higher trough levels are needed to prevent all bleedings, particularly during periods of vigorous activity. ${ }^{15}$ Pediatric patients present additional challenges compared with adult patients as this population is generally more dependent on a caregiver as well as being highly active. Prophylaxis with rIX-FP maintains a trough level of $>5 \%$ in pediatric patients across all dosing regimens and mean steady-state FIX activity levels were 15.1 and 7.2\% in patients on 7- and 14-day prophylaxis, respectively.

Other rFIX products have demonstrated similar results; mean steady-state FIX trough levels were $15 \%$ in patients aged 0 to 6 years and $19 \%$ in patients aged 7 to 12 years on once-weekly $40 \mathrm{IU} / \mathrm{kg}$ prophylaxis with N9-GP (Refixia, Novo Nordisk), a glycoPEGylated EHL-FIX. ${ }^{16}$ There have been no studies demonstrating steady-state FIX trough levels for pediatric patients treated with rFIXFC (ALPROLIX, Bioverativ); additionally, the phase 3 protocol does not define target trough levels during this study, and is thus difficult to compare with the data described here.

Both rIX-FP and rFIXFc have shown excellent tolerability, with no inhibitor development, no treatment-related AEs, or thromboembolic events in a pediatric population..$^{10,17}$ No inhibitor development or thromboembolic events have also been reported in pediatric patients receiving N9-GP ${ }^{16}$; however, the implications of long-term exposure to PEGylated products-especially from a young age-have not been established and therefore N9-GP is not currently indicated for prophylactic use in pediatric patients.

Prophylaxis with rIX-FP was highly effective in preventing bleeding episodes, demonstrated by low AsBRs across all regimens. There was a similar proportion of patients experiencing zero joint bleeding episodes across all dosing regimens; this is an important observation that should be considered when selecting patients who will benefit from extended dosing intervals. Additionally, the majority (50.4\%) of bleeding episodes were traumatic, consistent with bleeding characteristics seen in highly active pediatric populations; the phase 3 N9-GP trial in pediatric patients reported $61.9 \%$ of all bleeds were traumatic. ${ }^{16}$ rIX-FP demonstrated efficient treatment of bleeding episodes, with the majority of bleeding episodes $(96 \%)$ controlled with one or two infusions. ABRs for 7-day prophylaxis were comparable between the lead-in (median: 3.1 ; mean: 3.7 ) and extension (median: 2.0; mean: 3.3 ) studies, showing that rIX-FP provides stable bleeding protection at both early and late-phase weekly prophylaxis. Median (mean) ABRs reported in this pediatric cohort were higher than those reported in the adult cohort (2.0 [3.3] and 1.3 [2.5], respectively), ${ }^{11}$ which may reflect the higher activity levels of younger patients. rIX-FP 7-day prophylaxis had a comparable median (mean) ABR to another extended half-life FIX product, rFIXFc (initial weekly dose
$50-60 \mathrm{IU} / \mathrm{kg}$; subsequent dose to a maximum of $100 \mathrm{IU} / \mathrm{kg}$ or maximum frequency of twice weekly) and a slightly higher ABR than N9-GP (2.0 [3.3], 2.0, and 1.0 [1.44], respectively) 16,17; however, these studies were conducted in different patient populations and so these comparisons should be interpreted with caution.

Two patients transiently showed target joints during the extension study. This was unexpected considering that no target joints previously developed in pediatric patients in the lead-in phase 3 trial, and the relatively high trough levels reported during this study. However, trough levels were not measured immediately prior to or following these bleeding episodes; therefore, it is difficult to draw solid conclusions regarding the relationship of trough levels to these specific bleeds. Additionally, two of these target joints developed following a traumatic bleeding event and so were not necessarily correlated with reported trough levels. All reported target joints were well managed with dosing adjustments that allowed patients to continue with rIX-FP prophylaxis and at the end of the study, target joints were resolved in both patients. Comparable target joint incidence was identified during pediatric trials with rFIXFc and N9-GP, with no spontaneous bleeds reported in target joints during these studies $^{16,17}$; however, the nature or frequency of these target joints was not reported.

Dosing every 14 days with rIX-FP showed a comparable AsBR to 7-day prophylaxis in selected pediatric patients and might be considered an alternative treatment option for some patients. Extended dosing intervals of every 8 to 16 days with rFIXFc have been investigated in a small group of selected pediatric patients $(n=5) .{ }^{18}$ Median AsBR was 0.0 in the extended interval dosing regimen with rFIXFc $^{18}$; however, it must be considered that only patients aged 6 to 12 were eligible to participate in the extended interval dosing cohort, and so these data are not directly comparable to the rIX-FP extension study where younger patients could receive rIX-FP with extended dosing intervals of 10 or 14 days, at the investigators discretion, irrespective of their age. The dosing regimen was also adjusted per patient and the median dosing interval was 10 days with a median weekly dose of $66.6 \mathrm{IU} / \mathrm{kg}$, and so these results cannot be directly compared with the 14-day results with rIX-FP. Currently, there are no other published trials demonstrating an extended dosing interval of longer than 7 days with other rFIX products in pediatric patients. There are no head-tohead clinical studies comparing extended dosing intervals with rIX-FP and other extended half-life rFIX products in pediatric patients, making reliable comparisons in terms of efficacy and safety difficult.

\section{Conclusion}

This extension study has demonstrated the long-term safety and efficacy of rIX-FP prophylaxis in pediatric patients treated for up to 4 years. This study has also shown that extended intervals of 10 or 14 days are possible with rIX-FP in selected pediatric patients well controlled on a 7-day regimen while maintaining adequate efficacy. Reduced 
infusion frequency provides dosing flexibility and higher trough levels have the potential to improve long-term outcomes in pediatric patients with hemophilia B. Prophylaxis with rIX-FP represents a paradigm shift in the treatment of hemophilia B, setting a new standard of care.

\section{What is known about this topic?}

- Weekly prophylaxis with rIX-FP is effective for the prevention and treatment of bleeding episodes in children with hemophilia B.

- Compared with adult patients, pediatric patients have additional considerations when choosing a treatment regimen such as difficulty with venous access, a higher number of traumatic bleeds, higher FIX clearance and requirement for higher activity levels.

- Extended dosing intervals and reduced infusion frequency may decrease both patient and caregiver burden.

\section{What does this paper add?}

- The results of this study confirm the safety and efficacy of rIX-FP prophylaxis over approximately 4 years in pediatric patients with hemophilia $B$.

- Very low annualized spontaneous bleeding rates were achieved with dosing regimens up to 14 days.

- Extended regimens of 10 or 14 days are possible with rIX-FP in selected pediatric patients while maintaining safety and efficacy.

\section{Note}

Editorial support for the writing of this manuscript was provided by Jessica Jackson of Meridian HealthComms in accordance with good publication practice (GPP3) and was funded by CSL Behring. CSL Behring reviewed and provided feedback on the paper. The authors had full editorial control of the paper and provided their final approval of all content.

Members of the PROLONG-9FP Investigators Study Group Australia: Christopher Barnes, Royal Children's Hospital, Victoria; Julie Curtin, The Children's Hospital Westmead, NSW; Canada: Anthony Chan, McMaster Children's Hospital, Hamilton, ON; Czech Republic: Jan Blatny, Children's University Hospital, Masaryk University, Brno; Bohumir Blazek, University Hospital Ostrava, Ostrava-Poruba; Vladimir Komrska, University Hospital Motol, Prague; Germany: Hans-Jürgen Laws, Department of Pediatric Oncology, Hematology and Clinical Immunology, HeinrichHeine Universitaet, Dusseldorf; Spain: Maria-Teresa Álvarez Román, Hospital Universitario La Paz, Madrid.

Funding

This study was sponsored by CSL Behring.
Conflict of Interest

G.K.: Research grant support from Alnylam, BPL, Bayer, Baxalta, CSL Behring, Opko biologics, Pfizer, Shire; consultant for Alnylam, Bayer, Opko biologics, Pfizer, Shire, Roche; honoraria/speakers bureau for Bayer, CSL, Pfizer, Roche. H.C.: Research support from CSL Behring, LFB, Novo Nordisk, Octapharma, Shire/Takeda, SOBI; honoraria from Bayer, LFB, Octapharma, Pfizer, Roche, SOBI; travel support from Bayer, LFB, Octapharma, Pfizer, Roche, Sobi; C.M.: Research support from Bayer, Biotest, CSL Behring, Shire/Takeda; honoraria from Bayer, Biotest, CSL Behring, Novo Nordisk, Pfizer, Roche, Takeda; travel support from Biotest, Bayer, CSL Behring, Novo Nordisk. S.H.: received honoraria for speaking from Bayer Healthcare, Baxalta Innovation, Biotest, CSL Behring, Novartis Pharma, Novo Nordisk, Octapharma, Pfizer; research grants from Bayer Healthcare, Baxalta, Biotest, CSL Behring, Novo Nordisk, Octapharma, Pfizer; T.L.: Research grant support and honoraria from Bayer, CSL Behring, Novo Nordisk, Octapharma, Pfizer, Roche, Shire, SOBI; honoraria from Uniqure; Y.L. and W.S. are employees of CSL Behring; E.S.: Research grant support from CSL Behring; honoraria for speaking and/or for consulting from Bayer, Bioverativ, CSL Behring, Grifols, Kedrion, Novo Nordisk, Octapharma, Pfizer, Roche, Shire/Takeda, SOBI, Spark, Uniqure.

\section{Acknowledgments}

The authors would like to thank the PROLONG-9FP clinical study investigators for their contribution.

\section{References}

1 Srivastava A, Brewer AK, Mauser-Bunschoten EP, et al; Treatment Guidelines Working Group on Behalf of The World Federation of Hemophilia. Guidelines for the management of hemophilia. Haemophilia 2013;19(01):e1-e47

2 Konkle BA, Huston H, Nakaya Fletcher S. Hemophilia B. Seattle, WA: GeneReviews(R); 1993

3 Bolton-Maggs PH, Pasi KJ. Haemophilias A and B. Lancet 2003;361 (9371):1801-1809

4 Clausen N, Petrini P, Claeyssens-Donadel S, Gouw SC, Liesner R; PedNet and Research of Determinants of Inhibitor development (RODIN) Study Group. Similar bleeding phenotype in young children with haemophilia A or B: a cohort study. Haemophilia 2014;20(06):747-755

5 den Uijl IE, Fischer K, Van Der Bom JG, Grobbee DE, Rosendaal FR, Plug I. Analysis of low frequency bleeding data: the association of joint bleeds according to baseline FVIII activity levels. Haemophilia 2011;17(01):41-44

6 Shapiro A, Potts J, Li S, et al. Association of bleeding tendency with time under target FIX activity levels in severe hemophilia B patients treated with recombinant factor IX Fc fusion protein. Blood 2013;122(21):2349-2350

7 Castaman G. The benefits of prophylaxis in patients with hemophilia B. Expert Rev Hematol 2018;11(08):673-683

8 Santagostino E, Mancuso ME. Barriers to primary prophylaxis in haemophilic children: the issue of the venous access. Blood Transfus 2008;6(Suppl 2):s12-s16

9 Metzner HJ, Weimer T, Kronthaler U, Lang W, Schulte S. Genetic fusion to albumin improves the pharmacokinetic properties of factor IX. Thromb Haemost 2009;102(04):634-644

10 Kenet G, Chambost H, Male C, et al; PROLONG-9FP Investigator Study Group. Long-acting recombinant fusion protein linking 
coagulation factor IX with albumin (rIX-FP) in children. Results of a phase 3 trial. Thromb Haemost 2016;116(04):659-668

11 Mancuso M, Lubetsky A, Pan-Petesch B, et al. Long-term safety and efficacy of rIX-FP prophylaxis with dosing intervals of 7,10 , 14 and 21 days in previously treated adults and adolescents with hemophilia B. J Thromb Haemost 2020 [in press]

12 World Medical Association. World Medical Association Declaration of Helsinki: ethical principles for medical research involving human subjects. JAMA 2013;310(20):2191-2194

13 Verbruggen B, Novakova I, Wessels H, Boezeman J, van den Berg M, Mauser-Bunschoten E. The Nijmegen modification of the Bethesda assay for factor VIII:C inhibitors: improved specificity and reliability. Thromb Haemost 1995;73(02):247-251

14 Santagostino E, Martinowitz U, Lissitchkov T, et al; PROLONG-9FP Investigators Study Group. Long-acting recombinant coagulation factor IX albumin fusion protein (rIX-FP) in hemophilia B: results of a phase 3 trial. Blood 2016;127(14):1761-1769
15 Jiménez-Yuste V, Auerswald G, Benson G, et al. Achieving and maintaining an optimal trough level for prophylaxis in haemophilia: the past, the present and the future. Blood Transfus 2014;12 (03):314-319

16 Carcao M, Zak M, Abdul Karim F, et al. Nonacog beta pegol in previously treated children with hemophilia B: results from an international open-label phase 3 trial. J Thromb Haemost 2016;14 (08):1521-1529

17 Fischer K, Kulkarni R, Nolan B, et al. Recombinant factor IX Fc fusion protein in children with haemophilia B (Kids B-LONG): results from a multicentre, non-randomised phase 3 study. Lancet Haematol 2017;4(02):e75-e82

18 Pasi KJ, Fischer K, Ragni M, et al. Long-term safety and efficacy of extended-interval prophylaxis with recombinant factor IX FC fusion protein (rFIXFc) in subjects with haemophilia B. Thromb Haemost 2017;117(03):508-518 